\title{
Die ökologischen Beziehungen stoffwechselnder Systeme vom Standpunkt der Regelungstheorie
}

\author{
W OLFGANG WIESER \\ II. Zoologisches Institut der Universität Wien, Österreich
}

\begin{abstract}
The ecological relations of metabolizing systems from the view point of cybernetics. An attempt has been made to classify the patterns of change that occur in metabolizing biological systems under the influence of environmental changes. It was found useful to distinguish between three types of changes: a) control: the system responds to environmental changes by analogous changes of one or several of its variables. b) homoeostas is: the system responds to environmental changes by activation of compensating mechanisms which tend to minimize the deviation of the system from a given state. c) "switching" phenomena: the system responds to environmental changes by the activation of new processes which cause the system to move from one state into another state. - It is emphasized that the presently known mechanisms of cellular regulation (regulation of gene activity, end-product inhibition, enzymatic regulation) in combination with nervous and hormonal factors suffice to suggest a few, albeit crude, models for the explanation of adaptive phenomena in organisms. To improve these models the interactions between components of living systems as well as the transmission and integration of information on the cellular level will have to be studied in more detail.
\end{abstract}

\section{EINLEITUNG}

In biologischen Experimenten spielen lebendige Systeme - das sind Organismen und Kulturen - fast immer die Rolle von "black boxes". Der Experimentator beobachtet, mißt Veränderungen in der Umwelt und registriert Veränderungen im System. Aus dem Beziehungsgefüge zwischen input und output schließt er auf Vorgänge im Inneren der "box". In besonderem Maße gilt dies für den physiologischen OKkologen, der die Anpassungen von Organismen an Umweltsvariable studiert. Manchmal kann das System aufgebrochen und Einzelprozesse können in relativer Isolation studiert werden; etwa Enzyme in ihrer Abhängigkeit von Temperatur- und anderen Anderungen.

In beiden Fällen: beim Studium von Einzelprozessen und bei der Analyse komplexer Organismen, besteht das Rohmaterial der Beobachtungen aus Veränderungen von Variablen. Das Ziel des Experimentators ist es, aus den gesetzmäßigen Beziehungen zwischen einzelnen Variablen Modellvorstellungen abzuleiten, die das Verhalten der Organismen unter dem Druck von Umweltsbedingungen mit immer größerer Genauigkeit beschreiben. Eine Ubersicht über die ökologisch bedingten Veränderungen lebender Systeme ist schwerlich durch eine Aufzählung aller spezifischen Reaktionen 
zu erreichen. Die Zahl der mechanischen, elektrischen oder chemischen, der anatomischen, histologischen oder physiologischen Veränderungen, durch die Organismen Veränderungen in ihren jeweiligen Umwelten beantworten, ist Legion, ihre Klassifizierung Gegenstand von Lehrbüchern über vergleichende Physiologie.

Ein möglicher Weg zu einer Übersicht besteht jedoch darin, nicht nach dem Inhalt, sondern nach der Form von Veränderungen zu fragen; nicht nach den spezifischen Bestimmungsstücken sämtlicher Reaktionsmechanismen, sondern nach ihren Verknüpfungsgesetzen. Wenn wir uns vergegenwärtigen, über welche Veränderungen lebender Systeme auf einem Symposium wie diesem hier gesprochen wird, dann glaube ich drei Kategorien unterscheiden zu können:

1. Das System reagiert auf die Veränderung einer Umweltsvariablen mit der analogen Veränderung einer biologischen Variablen. Wir wollen in so einem Fall von Steuerung sprechen.

2. Im System wirkt sich die Veränderung einer Umweltsvariablen als "Störung “ aus, durch die Vorgänge in Gang gesetzt werden, die die Kompensierung der Störung und das Wiederherstellen des "Sollzustandes" zum Ziele haben. Dies bezeichnen wir als Regelung.

3. Das System reagiert auf die Veränderungen einer Umweltsvariablen mit dem Ingangsetzen neuer, oft komplexer Systemprozesse. Derartige Veränderungen wollen wir dem Begriff Umschaltung subsumieren.

Diese drei Kategorien repräsentieren natürlich nicht isolierte Mechanismen; vielmehr sind sie eng miteinander verknüpt. Regelungsvorgänge beruhen auf der Steuerung bestimmter Variablen durch andere Variable, und was wir hier unter , $\mathrm{Um}_{\mathrm{m}}$ schaltung" zusammenfassen, ist die Grundlage von Entwidklungs- und Differenzierungsprozessen, an denen. Stetuerungs- und Regelungsprozesse entscheidenden Anteil haben.

Ich beabsichtige nun, jede dieser drei Kategorien im Hinblick auf Veränderungen des Stoffwechsels lebender Systeme kurz zu diskutieren.

\section{STEUERUNG}

Die Steuerung ist die dem Experimentalbiologen geläufigste Veränderung eines lebenden Systems. Schwankungen der Temperatur, der Lichtintensität, des $\mathrm{pO}_{2}$, der Substratkonzentration usw. können analoge Veränderungen in der Intensität des Stoffwechsels bewirken. Die Form dieser Beziehung kann linear oder nicht-linear sein, sie kann sowohl in einfachen wie in komplexen Systemen geschehen, sie kann direkt oder indirekt sein. Beispiele hierfür sind in Abbildung 1 gegeben.

Direkt ist etwa die Beziehung zwischen Enzymkonzentration oder Substratkonzentration und Reaktionsgeschwindigkeit in Enzymsystemen (Abb. 1a). In den übrigen dargestellen Beispielen ist zwischen input (T'emperatur, $\mathrm{pO}_{2}$, Darmgewicht) und output (Q02) ein komplexes System, nämlich der stoffwechselnde Organismus, „dazwischengeschaltet". Dies drückt sich - unter anderem - in der größeren Streuung der Meßwerte aus (z. B. Abb. 1a und 1b). Trotzdem besteht auch in diesen Fällen zwischen input und output eine echte Steuerungsbeziehung. 
Die Steuerungsbeziehung wird durch die Steigung der Kurve und allenfalls durch den Punkt, an dem der Übergang zum „Plateau“ stattfindet, charakterisiert. Bei Einführung einer zweiten input-Variablen ändern sich meist die Charakteristika des Systems
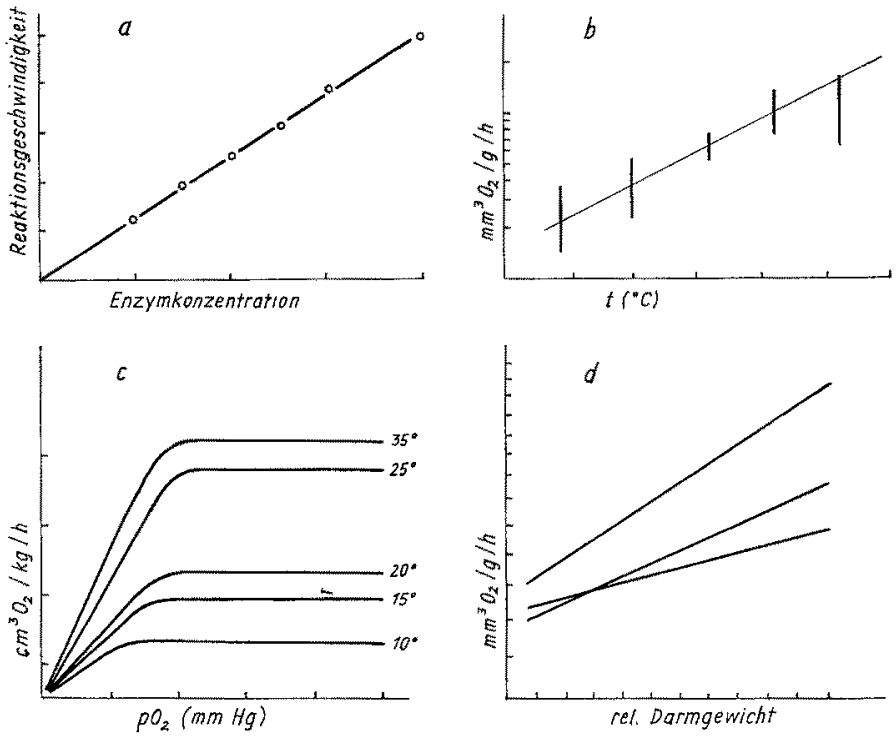

Abb. 1: Beispiele für Steuerungsvorgänge in biologischen Systemen. Die auf den Ordinaten aufgetragenen Variablen ändern sich in Abhängigkeit von Anderungen der Abszissen-Variablen. a: Abhängigkeit der Invertzudkerbildung von der Saccharase-Konzentration (nach BALDWIN 1952). b: Abhängigkeit des Q02 von der Temperatur bei Lumbricus terrestris (nach VALÈN 1958); die vertikalen Linien geben den Streuungsbereich der Meßwerte an. $c$ : Abhängigkeit des Qoz vom Sauerstoff-Partialdruck und der Temperatur bei Carassins carassius (nach FRY und HART aus Prosser 1950). $d$ : Abhängigkeit des Q02 vom relativen Darmgewicht bei Porcellio scaber; Regressionsgeraden sind für drei verschiedene Populationen dargestellt (nach WiESER 1963a)

(Abb. 1c). Es sei nochmals betont, daß wir es hier bloß mit einem Aspekt einer ökologischen Beziehung zu tun haben. So kann etwa die Intensivierung der Atmung bei Erhöhung der Temperatur gewissermaßen abstrakt für sich allein betrachtet werden. Andererseits kann die Erhöhung des $Q_{02}$ Ausdruck einer tiefgreifenden Richtungsänderung des Stoffwechsels sein und wird dadurch zum Element eines anderen Beziehungsgefüges.

\section{REGELUNG}

Die Veränderung eines Umweltfaktors kann als Störung aufgefaßt werden, die das System auszugleichen trachtet. Im black-box-Schema wäre dies so darzustellen, daß sich nach Veränderung des input der output zunächst im Sinne eines Steuerungsvorganges ändert, dann aber (und eventuell nach einigen nicht-linearen $Z$ wischenphasen) auf den Ausgangswert zurückfällt (Abb. 2). In der Literatur finden sich hierfür die Begriffe Anpassung, Homoeostasis oder Regelung, die einander zwar nicht kongruent 
sind, von denen aber jeder den oben dargestellten Fall enthält. Für die Funktionsweisen von Regelungsvorgängen gibt es seit einiger Zeit eine Theorie, die bisweilen unter dem Namen „Kybernetik“ läuft (WIENER 1948). Diese Theorie fordert unter anderem, daß bei Regelungsvorgängen die Veränderungen der konstant zu haltenden

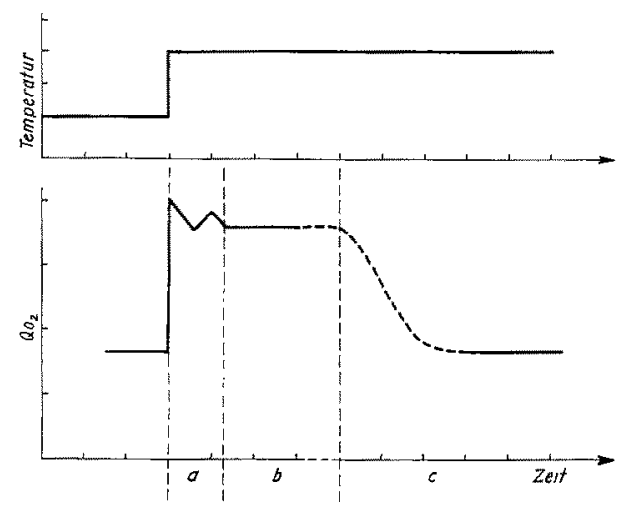

Abb. 2: Modellhafte Darstellung des Verlaufes der Sauerstoffverbrauchs-Kurve eines Organismus nach plötzlicher Temperatursteigerung. a: Exste Gleichgewichtsänderung des Systems ("overshoot"); $b$ : Steuerungsphase (Einstellung auf neues Gleichgewicht); $c$ : Regelungsphase (Rückkehr auf altes Gleichgewicht). $a$ und $b$ nach Angaben Grarngers (1958) für Artemia salina; $c$ gilt für alle Organismen, welche die Wirkungen plötzlicher Temperaturänderungen völlig kompensieren (Typ 2 nach PRECHT 1955)

Variablen einem Regler gemeldet werden, worauf der Regler Prozesse in Gang setzt, welche die Veränderungen des Systems zu kompensieren trachten. Der "Sollwert" muß auf irgendeine Wejse im Regler festgelegt sein. Die für den gesamten Funktionszusammenhang nötigen Elemente müssen auch in lebendigen Systemen überall dort vorhanden sein, wo Regelungsvorgänge nachweisbar sind (WIESER 1963b). Die Arbeitsweisen biologischer und technischer Regelsysteme weisen jedoch beträchtliche Unterschiede auf (CHANCE 1961). So beruht Rückkopplung in elektrischen Systemen auf der Umkehr eines Stromflusses ( + und - ), in biologischen hingegen auf dem Hinzufügen und Entfernen von Substanzen (antagonistisches Prinzip). Hierdurch werden Probleme eigener Art eingeführt (Goldman 1960).

Innerhalb gewisser Grenzen können die „störenden“ Wirkungen sämtlicher ökologischen Faktoren durch biologische Systeme ausgeglichen werden, wobei die Regelungsmöglichkeiten um so größer sind, je komplexer das System oder je höher organisiert das Lebewesen ist. So ändert sich z. B. die Atmung von Bakterien in direkter Abhängigkeit von der Substratkonzentration, während die Atmung höherer Organismen und ihrer Gewebe von der Substratkonzentration weitgehend unabhängig sein kann (BARRON 1958). In letzterem Fall hält also ein homoeostatischer Mechanismus den Stoffwechsel mehr oder minder konstant.

In ähnlicher Weise werden bei verschiedensten Organismen Parameter des Stoffwechsels trotz Anderungen der Umweltstemperatur, des Ionengleichgewichtes, des Wassergehaltes, des Ernährungszustandes usw. konstant oder annähernd konstant ge- 
halten (für Beispiele und Diskussionen siehe unter anderem Mrttelstaedt 1954, Hassenstein 1960, MCLean 1960, Goldman 1960).

Das Prinzip der Regelung findet sich auf allen Ebenen der biologischen Organisation. So läßt sich Temperaturregelung in Enzymsystemen (PRECHT 1951), in isolierten Geweben (Peiss \& Field 1950, Schlifeper 1952), poikilothermen (Bullock 1955) und natürlich in homoiothermen Organismen (HeNsEL 1955) nachweisen. Regulierende Mechanismen müssen also in subzellulären, zeilulären und multizellulären Systemen existieren. In mehrzelligen Organismen fungieren oft Nervensystem und Hormone als die wichtigsten Bestandteile von Regelkreisen. Ihre Wirkung und Verknüpfung ist oft genug diskutiert worden und braucht uns hier nicht weiter zu beschäftigen. In zellulären Systemen sind Regelungsmechanismen in letzter Zeit mehrfach beschrieben worden. Folgen wir der Übersicht von HoLzer (1963), dann wären als wichtigste derartige Mechanismen, die für die Regelung des Stoffwechsels von Bedeutung sein können, zu nennen:

\section{Regulation der Genaktivität}

Auf Grund der Verknüpfung von DNS, RNS und Proteinsynthese ist die Regulation der Genaktivität eine der Möglichkeiten, die Bildung von Enzymen zu kontrollieren. Nach JACOB \& MONOD (1961) hängt die Bildung eines spezifischen Enzyms von einer funktionellen genetischen Einheit, dem Operon, ab, dessen Aktivität durch ein Regulator-Gen kontrolliert wird. Im allgemeinen hemmt der Regulator die Synthese eines Enzyms. Ein rom Zellplasma in den Kern diffundierender Stoff kann jedoch die Hemmung autheben (De-Repression) und die Bildung des Enzyms induzieren. Das so gebildete Enzym mag den Stoffwechsel in eine neue Bahn lenken. Damit haben wir aber die Elemente eines Regelkreises vor uns: Eine Umweltsänderung bewirkt eine Stoffwechseländerung, was zur Anhäufung einer Substanz im Zellplasma führt. Die Substanz gelangt in den Kern, wo sie über DNS und RNS die Bildung eines Enzyms induziert. Das neugebildete Enzym greift in den Stoffwechsel ein und stellt das ursprüngliche Gleichgewicht wieder her.

\section{Endprodukthemmung}

Die Anhäufung einer Aminosäure oder eines Proteins hemmt meist die eigene Synthese, indem das Endprodukt die Aktivität eines Schlüsselenzyms in der SyntheseSequenz blockiert (UMBarger 1956, Davis 1961). Dies ist ein echter Rückkopplungsmechanismus, der sicherlich oft Bestandteil eines intrazellulären Regelsystems ist. So wird etwa nach Goriñ (1958) die Intensität der Arginin-Synthese bei E. coli durch die Konzentration von Arginin im Bakterium und im Medium genau geregelt.

\section{Enzymatische Regulation}

Die meisten Reaktionssysteme in Organismen hängen von der Konzentration kritischer Coenzyme oder Substrate ab. Durch Regelung der Konzentrationen dieser 
Schlüsselelemente kann das gesamte System reguliert werden. Das wichtigste Beispiel hierfür ist die Abhängigkeit der Atmungskettenphosphorylierung vom System

$$
\mathrm{P}+\mathrm{ADP} \leftrightharpoons \mathrm{ATP}
$$

Die Energie des Kohlenhydratabbaues kann nur dann genïtzt werden, wenn genug ADP für die ATP-Synthese vorhanden ist, und ADP wird meist durch den Abbau von ATP nachgeliefert. Eine Regelung der Atmungsintensität könnte also durch Steuerung der ADP-oder ATP-Konzentration erfolgen. LYNEN et al. (1959) vermuten auch, daß dies der entscheidende Mechanismus zur Regelung des Energiekreislaufes in der Zelle ist. Nach dieser Anschauung wird ATP je nach Bedarf dem Zellstoffwechsel entzogen oder zur Verfügung gestellt, wobei als Regler und Speicher die Mitochondrien fungieren. Die stoffwechselregelnde Funktion der Mitochondrien wird auch durch andere Untersuchungen nahegelegt. SMITH (1956) zeigte, daß Faktoren, die die aktive Masse der Mitochondrien verändern, auch den Sauerstoffverbrauch beeinflussen. Nach MuNDAY \& THOMPson (1962) soll die Konzentration der Körperflüssigkeit der Krabbe Carcinus maenas in hyposmotischen Medien so aufrechterhalten werden, daß die Mitochondrien zu schwellen beginnen, wodurch mehr ATP freigesetzt und die Atmungsintensität gesteigert wird. Die erhöhte Aktivität dient dazu, das überschüssige Wasser aus der Körperflüssigkeit hinauszupumpen. Damit ist die Steuerung des Qoz durch die osmotische Konzentration des Mediums Teil eines Regelkreises zur Aufrechterhaltung der osmotischen Konzentration der Körperflüssigkeit.

Diese Diskussion intrazellulärer Mechanismen sowie die Erinnerung an hormonale und nervöse Erscheinungen bei höheren Tieren dürfte deutlich machen, daß für den Aufbau homoeostatischer Systeme auf sämtlichen Lebensstufen eine Fülle funktioneller Elemente bereitstehen. Um dies za illustrieren, sei in Abbildung 3 der Mechanismus der Regelung von Stoffwechsel oder Körpertemperatur bei Erhöbung der Milieutemperatur in einem enzymatischen (a), einem intrazellulärem (b) und einem vielzelligen biologischen System (c) auf modellhafte Weise dargestellt.

In Beispiel a) führt die Temperaturerhöhung nach physikochemischen Gesetzen zu einer Reaktionsbeschleunigung und schließlich zur Anhäufung des Endproduktes. Die gesteigerte Konzentration des Endproduktes hemmt jedoch die eigene Bildung, indem es ein Schlïsselenzym (meist ein „branchpoint"-Enzym: Davis 1961) blockiert. Hierdurch kann die Reaktionsgeschwindigkeit mehr oder minder auf das ursprüngliche $\mathrm{Maß}$ reduziert werden. Oszillationen sind in so einem System nicht zu vermeiden.

In Beispiel b) ist die Situation bereits komplexer, da zwei mögliche Regelungssysteme zur Verfügung stehen, die Mitochondrien im Zellplasma und die Gene im Zellkern. Die Erhöhung der Stoffwechselintensität auf Grund der Temperaturerhöhung führt zù einer Beschleunigung des Umsatzes im ADP/ATP-System. Wird nun etwa ATP in den Mitochondrien gebunden, dann stehen weniger ATP-Moleküle zur Energiegewinnung und dementsprechend weniger ADP-Moleküle für die Atmungskettenphosphorylierung zur Verfügung. Damit muß aber die Stoffwechselintensität sinken. Eine zweite Regelmöglichkeit läge darin, daß über den Kern eine Neueinstellung des enzymatischen Gleichgewichtes erfolgen kann. Dies würde die oben erwähnte (S. 360) Regulation der Genalktivität implizieren. Die Tatsache, daß es bei poikilothermen Tieren schnelle und langsame Regulationsprozesse gibt, die ziemlich scharf voneinander getrennt zu sein scheinen (Bullock 1955, S. 321), mag ein Ausdruck der 
Existenz dieser zwei zellulären Regelkreise sein, wobei natürlich die "MitochondrienRegelung" die schnellere, die „Gen-Regelung" die langsamere wäre. Verallgemeinerungen sind hier jedoch noch sehr unsicher. Außerdem ist die Möglichkeit einer zentralen Regelung uiber das Nervensystem in Betracht zu ziehen (Roots \& PRosser 1962).
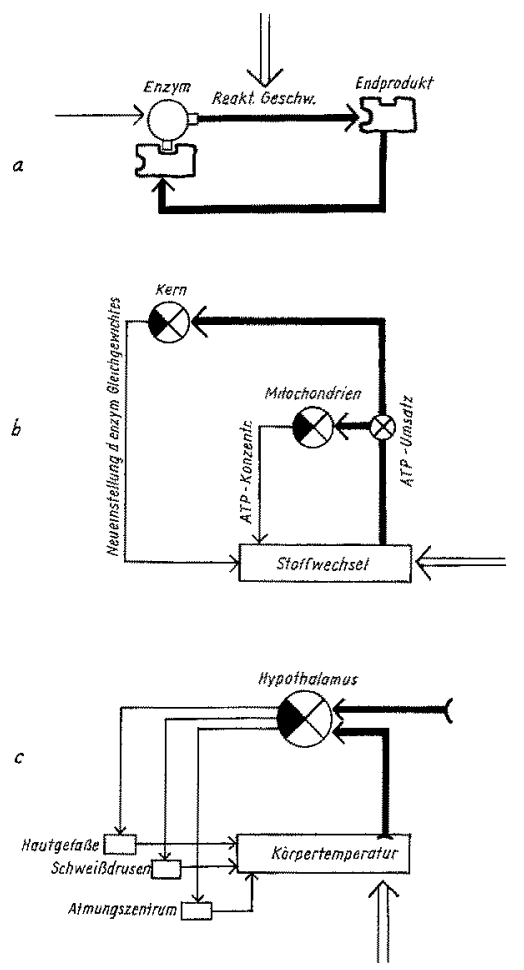

Abb. 3: Blockschaltbilder zur schematischen Darstellung des Funktionszusammenhanges bei Regelungsvorgängen nach Erhöhung der Außentemperatur in subzellulären (a), zellulären (b) und multizellulären (c) Systemen. Die Störgröße ist durch den Doppelpfeil angedeutet. Sie stevert die durch starke schwarze Pfeile gekennzeichneten System-Variablen (Reaktionsgeschwindigkeit, ATP-Umsatz, Impulsfrequenz, Hormonkonzentration), die ïber die Regler (Kreise) die Kompensation des "Stör"effektes bewirken. Halbkreise in 3c stellen Rezeptoren, kleine Rechtecke Effektoren dar. Die großen Rechtecke in $3 b$ und $c$ repräsentieren die konstant zu haltende Regelgröße, der in $3 a$ die Konzentration des Endproduktes entsprechen würde

Beispiel c) abstrahiert nur einige bekannte Tatsachen über die Regelung der Körpertemperatur bei Säugetieren. Von Bedeutung ist, daß hier - zum Unterschied von Beispiel a) und b) - die Körpertemperatur und nicht die Stoffwechselintensität als Regelgröße fungiert, obwohl kaum daran zu zweifeln ist, daß eine der Hauptfunktionen der Homoeothermie die Schaffung eines Milieus ist, in dem Prozesse des Stoffwechsels mit konstanter Geschwindigkeit ablaufen können. Da aber die Konstanthaltung der Körpertemperatur Energie erfordert, müssen zur Aufrechterhaltung der Stoffwechselkonstanz Stoffwechselenergien abgezweigt werden - eine nur scheinbar paradoxe Situation, welche die steigende Differenzierung der Organismen auf der Evolutionsskala illustriert. 
Regelungssysteme dieser Art tragen alle zur Autonomie des Stoffwechsels vom Diktat der Umwelt bei. Dies können sie aber nur dann wirklich erfolgreich, wenn sie mit anderen Regelungssystemen verknüpt sind. Eine der wichtigsten Voraussetzungen für das Funktionieren eines homoeostatischen Mechanismus wie dem der Temperaturregelung muß - so sollte man meinen - adäquate Versorgung mit Energie selbst bei variablen Nahrungsverhältnissen sein. Dies trifft auch für die meisten Warmblüter zu. Bei erwachsenen höheren Säugern z. B. funktioniert die Temperaturregelung selbst nach längeren Hungerperioden. Bei Jungtieren, primitiven Säugern und einigen Vögeln hingegen arbeitet der Mechanismus nicht mit demselben Grad der Perfektion. So wies etwa LAsIEwsKI (1963) nach, daß Kolibris nur dann homoeotherm sind, wenn sie über genügend Energiereserven verfügen. Bei geringen Reserven wird der homoeostatische Mechanismus abgebaut, und das Tier fällt in einen Zustand der Starre. Hier ist also unter anderem wegen der Kleinheit und hohen Aktivität der Tiere - eine ausreichende Versorgung der Regelungsmechanismen mit Energie nicht unter allen Umständen möglich - und dieser "Unvollkommenheit" muß in der Organisation der Art Rechnung getragen sein.

Die Verwendung des Wortes "Unvollkommenheit" in diesem Zusammenhang mag von manchen als eine teleologische Abweichung von der Parteilinie der streng kausalen und zweckfreien biologischen Forschung gedeutet werden. Im Begriffssystem der Regelungstheorie hat jedoch die Vorstellung eines Zieles, das auf mehr oder minder vollkommene Weise erreicht wird, ihren legitimen Platz. Das Ziel ist die Aufrechterhaltung eines bestimmten Gleichgewichtszustandes. Diese Deutung haben schon ROSENBLÜtH, WIENER \& Bigelow (1943) yor 20 Jahren gegeben. So können wir auch sagen, daß gewisse poikilotherme aquatische Tiere eine nur "unvollkommene Temperaturanpassung des Q02 zeigen (Scholander, Flagg, Walters \& IRving 1953), oder daß der Entwicklungszyklus von Sagitta elegans nur „unvollkommen“ an die tiefen Temperaturen des arktischen Wassers angepaßt ist (Dunbar 1962), oder daß das Wachstum litoraler Mollusken im Vergleich zum Energieverbrauch nur „unvollkommen" an Temperaturunterschiede angepaßt ist (DEHNEL 1955).

Derartige Insuffizienzen physiologischer Regelungsmechanismen dürften von groBer ökologischer Bedeutung sein, da sie die Voraussetzungen beeinflussen, unter denen die betroffenen Organismen zu leben imstande sind.

\section{UMSCHALTUNG}

Nicht alle Veränderungen biologischer Systeme lassen sich den Begriffen „Steuerung" und "Regelung" subsummieren. Zahlreiche Leistungen von Organismen sind dadurch gekennzeichnet, daß auf Grund einer Veränderung in der Umwelt gänzlich neue Prozesse in Gang gesetzt werden. Bietet man etwa Individuen der Isopoden-Art Porcellio scaber nach einer Reihe von Kurztagen (7 Stunden Licht, 17 Stunden Dunkelheit) eine Reihe von Langtagen (16 Stunden Licht, 8 Stunden Dunkelheit), dann ändert sich zunächst die stark proportionale Beziehung $z$ wischen dem Darmgewicht und dem Sauerstoffverbrauch (Abb. 1d). Letzterer wird weitgehend unabhängig vom Ernährungszustand des Tieres (Abb. 4). Dies deutet auf das Einsetzen neuer Stoff- 
wechselvorgänge, die schließlich zur Gonadenreifung und zur Fortpflanzung der Tiere führen. Die Feststellung, die Hell-Dunkel-Periodik "steuere“ das Fortpflanzungsgeschehen, wird den Tatsachen nicht ganz gerecht. Im Grunde handelt es sich um eine tiefgreifende Umschaltung des Stoffwechsels der Art unter dem Einfluß des Lichtes.

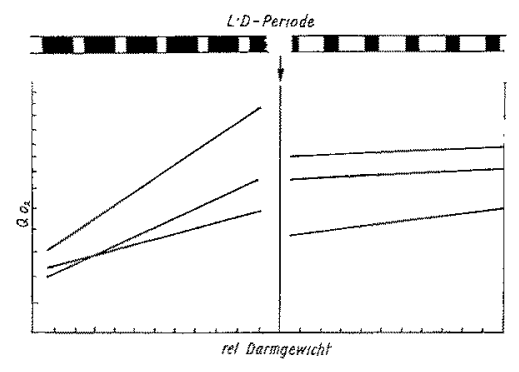

Abb. 4: Der Einfluß der Licht-Dunkel-Periodik auf die Beziehung zwischen Q02 und relativem Darmgewicht bei Porcellio scaber. Regressionsgeraden sind für drei Populationen eingezeichnet. Linker Abschnitt: 7L:17 D, rechter Abschnitt: 16L :8D (nad. WIEser 1963a)

In ähnlicher Weise kann der Ubergang vom Kurztag zum Langtag bei Pflanzen Blütenbildung hervorrufen, kann Sauerstoffmangel die Umschaltung vom aeroben zum anaeroben Stoffwechsel bewirken, kann ein Nahrungsfaktor Häutungsprozesse einleiten, das Erreichen einer kritischen Temperatur die Entwicklung eines Eies in Gang setzen usw. Derartige Beziehungen müssen in einer allgemeinen Theorie von der Umweltsabhängigkeit lebendiger Systeme ebenfalls ihren Platz finden.

Dies verursacht keine besonderen Schwierigkeiten, da ja bereits bei Besprechung der zellulären Regelung angedeutet wurde, daß unter dem Einfluß von Außenfaktoren Enzymkonzentrationen geändert und damit Stoffwechselvorgänge in neue Bahnen gelenkt werden können. Am dramatischsten ist diese Umschaltmöglichkeit in zellulären Systemen wohl durch die Entdeckung von ,induzierbaren Enzymen“ (Monod 1956, Davis 1961) demonstriert worden.

Wie das Prinzip der Regelung ist auch das der Umschaltung auf allen Ebenen der biologischen Organisation möglich. So kann in Krebszellen der anaerobe Kohlenhydrat-Abbau durch den aeroben ersetzt werden, wenn die Konzentration des

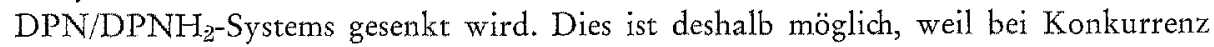
um dieses System die Enzyme der Atmung gegenüber denen der Glykolyse den Vorzug haben (Holzer 1963). Da nun einige als Cytostatica bekannte Drogen die Konzentration des DPN/DPNHq-Systems zu senken scheinen. (HolzkR 1961), können wir für diesen Fall sagen, daß ein Außenfaktor durch Beeinflussung eines enzymatischen Schlüsselsystems die Umschaltung zellulärer Prozesse von anaerob auf aerob bewirken kann.

Auf der nächsten Stufe der Komplexität spielen sich Umschaltungen des Stoffwechsels unter der Mitwirkung von Genen ab, die unter dem Begriff "Regulation der Genaktivität" bereits erwähnt wurden (S. 360): Durch ein neues Substrat kann die Aktivität eines "Operon" in Gang gesetzt und damit die Bildung eines neuen Enzyms induziert werden.

An dieses Modell schließs sich der von Clever \& Karlson (1960) analysierte 
Fall der Wirkung des Hormons Ecdyson auf das Einsetzen der Häutung bei Cbironomus an. Das Hormon wirkt auf einen charakteristischen Locus (1 R 18) im Genom der Diptere, von dem - zweifellos via RNS und Enzyme - Informationen ausgehen, die die tjefgreifenden metabolischen Veränderungen des Häutungsvorganges initiieren. Dieses Beziehungssystem läßt sich ganz mit den Begriffen der Substratinduktion beschreiben, wenn das Hormon Ecdyson als das induzierende Substrat aufgefaßt wird.

Diese Reaktionskette kann noch um eine Stufe in die Umwelt hinaus verlängert werden. Die Ausschüttung von Hormonen oder neurosekretorischen Substanzen wird meist durch Umweltsfaktoren wie Licht oder Temperatur gesteuert, so daß sich für metabolische Schaltprozesse folgende Sequenz ergibt:

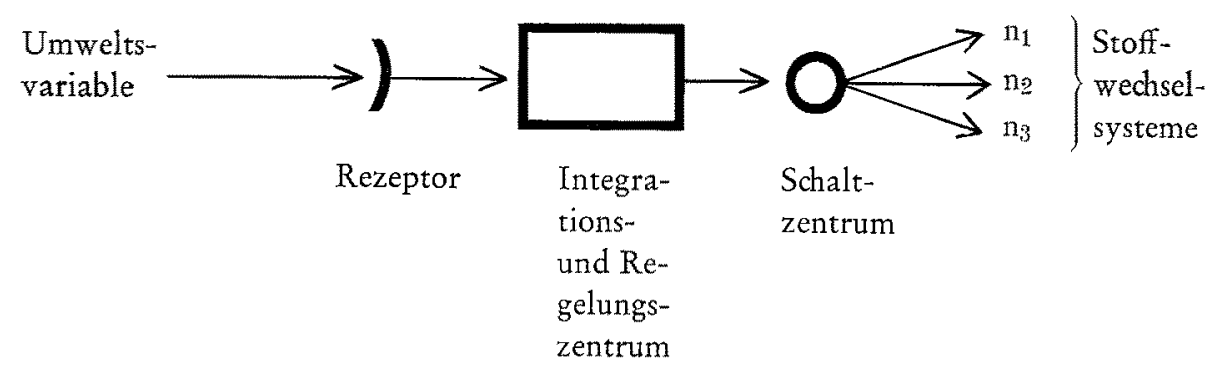

Dies ist natürlich eine sehr schematische Darstellung. In Wirklichkeit ist die Vey knüpfung Umwelt-Stoffwechsel nicht als eine lineare Sequenz, sondern als ein Gefüge zu denken. Jedes Glied der Kette steht wieder mit anderen Gliedern in Verbindung und kann durch diese beeinflußt werden. Hieraus entwickeln sich - in der Terminologie von Ashby (1960) - „multistabile Systeme“.

Als Beispiel sei etwa die bereits erwähnte Abhängigkeit des Fortpflanzungsgeschehens vom Erreichen einer kritischen Tageslänge im Friihling oder Herbst zitiert (BüNnING 1963, GIESE 1959, WIESER 1963a). Zusätzlich hängt der Ablauf dieses Geschehens nämlich auch noch von anderen Faktoren $a b$, die alle in den Kreis der Betrachtung gezogen werden müssen, will man das Beziehungsgefüge des gesamten Vorganges darstellen. So muß etwa eine kritische Temperaturschwelle überschritten worden sein, damit die vorbereitenden Stoffwechselprozesse überhaupt anlaufen können (ORTON 1920, WIEsER 1963a); die erhöhte Temperatur muß für eine gewisse Zeit geherrscht haben, um auf den Organismus wirken zu können (bei Ostrea edulis z. B. mehrere Wochen: Korringa 1957). Des weiteren können Phasen des Fortpflanzungsgeschehens von der Anwesenheit kritischer Nahrungsfaktoren abhängen (BARNes 1957 für Balanus balanoides, STRANGWAys-Dixon 1961 für Calliphora erythrocephala).

Wie in diesen (und vielen anderen) Fällen Licht, Temperatur, Nahrung und das Stoffwechselgeschehen miteinander verknüpft sind, ist das entscheidende Problem, an das der Experimentalbiologe ein bißchen mit der Einstellung eines Technikers heranzugehen hat, der die Schaltung einer - nicht von ibm entworfenen - komplizierten Maschine zu entwirren trachtet.

Neben der Aufklärung des „Wirkungsgefüges“ (MrtTelstaedt 1961) gibt es in diesem Zusammenhang noch ein zweites Hauptproblem, und das ist die Frage nach 
der Informationsverarbeitung im Organismus. Auf welche Weise initiiert etwa ein Umweltsreiz wie die Tageslänge oder die Wassertemperatur eine meßbare biologische Reaktion? Wie setzt eine neurale oder humorale Information einen Stoff wechselprozeß in Gang? Wir haben in den letzten Jahren eine Fülle relevanter Tatsachen erfahren, so etwa, daß Licht und Temperatur auf Integrationszentren (Schilddrüse, Corpora allata, spezifische Gehirnzellen) wirken, dort die Bildung von Hormonen oder Neurohormonen veranlassen, die bei Erreichen einer kritischen Schwelle in den Zielorganen Stoffwechseländerungen hervorrufen (MORGAN \& FALEs 1942 für Triturus viridescens, Roberts 1961 für Carassius carassius, Jungstand 1962 für Helix pomatia etc.). Uber die Einzelheiten der hier wirksamen Mechanismen sind wir jedoch noch weitgehend im unklaren. Das einzig Sichere dürfte sein, daß jeder ökologisch-physiologische Zusammenhang in das dynamische Wechselspiel von Genen, Ribosomen, Mitochondrien und Membranen, von DNS, RNS und Proteinen hineinverflochten ist.

In formaler Hinsicht lassen sich nun aber auf Grund der Eigenschaften der untersuchten Systeme einige Aussagen über die möglichen Formen der Informationsverarbeitung machen. Hier kehren wir zum Problem der Steuerung zurück, denn jeder Rezeptor, jedes Schaltelement kann als ein Steuerkörper (oder Informationswandler) aufgefaßt werden, der ein Signal oder eine Information empfängt, verarbeitet und als "verschlüsselte" Information weitergibt. Die „input-Information" steuert somit die "output-Information" (vgl. RankE-KeIdel 1960, Hassenstein 1960, Goldman 1960).

In Zusammenhang mit Stoffwechselphänomenen lassen sich vier Informationswandler unterscheiden: Vorzeichenwandler, Proportionalwandler, Differentialwandler und Integralwandler.

\section{Vorzeichenwandler (Zweipunktwandler)}

Der output hat nur zwei Zustände $(+,-)$, die durch den jeweiligen input bestimmt werden. Sämtliche Alles-oder-Nichts-Vorgänge gehören hierher. Klassisches Beispiel ist die Entladung eines Neurons. Die Aktivität eines Operons - und damit eines zugeordneten Enzyms - kann vielleicht ebenfalls nach diesem Schema gedeutet werden, denn es kennt nur zwei Zustände: Hemmung und Enthemmung. Die Entscheidung zwischen diesen beiden Zuständen hängt von der Aktivität des Regulator-Gens ab (JACOB \& Monod 1961).

\section{Proportionalwandler}

Die Größe der output-Variablen ist der Größe der input-Variablen proportional. So mag die Erhöhung der Temperatur zu einer proportionalen Erhöhung des ATPUmsatzes und damit des Q02 führen. Kommt es dann durch die regulative Wirkung der Mitochondrien zu einer proportionalen Verminderung der ATP-Konzentration, sprechen wir von einer Proportionalregelung.

Zahlreiche Stoffwechseländerungen können auf ähnliche Weise den Veränderungen von Enzymkonzentrationen proportional sein, letztere können auf Veränderungen hormonaler Faktoren ansprechen und diese sich in proportionaler Abhängigkeit von einer Umweltsgröße verändern. 


\section{Differentialwandler}

Die output-Variable hängt ab von der Geschwindigkeit (dem ersten Differentialquotienten), mit dem sich die input-Variable ändert. So dürfte es in der Regelung des Blutzuckergehaltes Rezeptoren geben, die auf die Geschwindigkeit, mit der sich die Zuckerkonzentration ändert, ansprechen (GoldDMAN 1960).

Der von Grainger (1958) und anderen beschriebene "overshoot" des Sauerstoffverbraùches hängt ebenfalls vom Differential der Unweltsänderung $a b$, da er nur bei raschem Temperaturwechsel deutlich ist. Ob die Folgen rascher Temperaturänderung, wie sie etwa von Loosanoff \& Davis 1952 für Crassostrea virginica beschrieben werden, hiermit in Beziehung stehen, ist unklar.

\section{Integralwandler}

Der output hängt davon $a b$, wie lange der input, ohne seine Größe zu ändern, einwirkt (also vom Zeitintegral des input). So muß eine kritische Temperatur meist eine gewisse Zeit geherrscht haben, damit bei Tieren Brutverhalten ausgelöst wird (Korringa 1957, WIESER 1963a), und dasselbe gilt für die Beziehung zwischen der Tageslänge und dem Einsetzen von Blütenbildung, Gonadenreifung etc. Genaugenommen, müßte man hier aber von einer Integral-Zweipunkt-Steuerung sprechen, da der output meist eine von zwei Möglichkeiten darstellt (siehe auch Ranke-KerDeL 1960). Eine echte Integralbeziehung dürfte jedoch zwischen der Tageslänge und der Bildung des neurosekretorischen Materials bestehen (JungsTand 1962).

Durch weitere Verfeinerung dieses Schemas könnte man vielleicht zu einer Klassifizierung aller Formen der Informationsverarbeitung in stoffwechselnden biologischen Systemen kommen. Der Auflösbarkeit setzt jedoch die Komplexität der meisten Systeme deutliche Grenzen.

Zusammenfassend läßt sich sagen, daß die Aufklärung spezifischer Beziehungen in lebenden Systemen die Grundlage aller biologischen Forschung ist, daß unsere Bemühungen aber unvollkommen bleiben müssen, wenn wir nicht versuchen, aus den individuellen Bestimmungsstücken jene höheren Verknüpfungssysteme aufzubauen, die die Voraussetzung aller Lebenserscheinungen sein müssen. Hierfür ist die quantitative Erfassung spezifischer Beziehungen - der Gegenstand dieses Symposiums unabdingbare Voraussetzung.

\section{ZUSAMMENFASSUNG}

1. Es wurde ein Versuch gemacht, die Veränderungen, die sich in stoffwechselnden Systemen unter dem Einfluß von Umweltänderungen abspielen, zu klassifizieren. Dabei erwies sich als günstig, zwischen drei Formen der Veränderung zu unterscheiden, nämlich: a) Steuerung: das System reagiert auf Umweltsänderungen mit analogen Veränderungen einer oder mehrerer seiner Variablen; b) R egelung: das System reagiert auf Umweltsänderungen mit der Aktivierung kompensierender Mechanismen, die die Abweichung des Systems von einem Sollzustand so gering wie 
möglich zu halten trachten; c) Umschaltung: das System reagiert auf Umweltsänderungen mit der Aktivierung neuer Prozesse, die die Uberführung des Systems yon einem Zustand in den nächsten bewirken.

2. Die bisher bekannt gewordenen Mechanismen zellulärer Regulation erlauben in Verbindung mit nervösen und hormonalen Faktoren die Formulierung einiger Modelle zur Erklärung von Anpassungserscheinungen bei Organismen.

3. Eine Verbesserung dieser Modelle bedarf eines genaueren Studiums der Verknüpfungsweisen der Bauelemente lebendiger Systeme sowie der Obertragung und Verarbeitung von Information auf zellulärer Ebene.

Herrn Professor B. Hassenstern, Freiburg, danke ich für kritische Anmerkungen zum Manuskript. Etwaige Ungenauigkeiten oder Fehler gehen jedoch allein auf das Konto des Autors.

\section{ZITIERTE LITERATUR}

Ashby, R., 1960. Design for a Brain. $2^{\text {nd }}$ ed. John Wiley \& Co., New York, 286 pp.

BuLDWIN, E., 1952. Dynamic Aspects of Biochemistry. Cambridge Univ. Press, 544 pp.

BARNes, H., 1957. Processes of restoration and synchronization in marine ecology. Ann. biol., Paris, 3. ser. 33, 67-85.

Barron, G. E. S., 1958. The regulatory mechanisms of cellular respiration. In: Perspectives in marine biology (edited by A. A. Buzzati-Traverso). Univ. of California Press, pp. $211-232$.

Bullock, T. H., 1955. Compensation for temperature in the metabolism and activity of poikilotherms. Biol. Rev. 30, 311-342.

Bünning, E., 1963. Die physiologische Uhr. 2. Aufl. Springer, Berlin-Göttingen-Heidelberg, $153 \mathrm{pp}$.

CHANCE, B., 1961. Control characteristics of enzyme systems. Cold Spr. Harb. Symp. quant. Biol. 26, 289-299.

Clevver, U. \& Karison, P., 1960. Induktion yon Puff-Veränderungen in den Speicheldrüsenchromosomen yon Chironomus tentans durch Ecdyson. Exp. Cell Res. 20, 623-626.

Davis, B. D., 1961. The teleonomic significance of biosynthetic control medranisms. Cold Spr. Harb. Symp. quant. Biol. 26, 1-10.

DeHnel, P. A., 1955. Rates of growth of gastropods as a function of latitude. Pbysiol. Zoöl. 28, 115-144.

Dunbar, M. J., 1962. The life cycle of Sagitta elegans in arctic and subarctic seas. J. Mar. Res. 20, 76-91.

GIESE, A. C., 1959. Comparative physiology: annual reproductive cycles of marine invertebrates. Annu. Rev. Physiol. 21, 547-576.

Goldman, S., 1960. Cybernetic aspects of Homeostasis. In: Mineral Metabolism. (edited by C. L. Comar \& F. Bronner). vol. I, Part A, Academic Press, New York and London, pp. 61-100.

GorrNr, L., 1958. Regulation en retour (feedback control) de la synthése de l'arginine chez Eschericbia coli. Bull. Soc. Chim. biol., Paris 40, 1939-1952.

Grannger, J. N. R., 1958. First stages in the adaptation of poikilotherms to temperature change. In: Physiological adaptation (edited by C. L. Prosser) Am. Physiol. Soc. Washington, D. C., pp. 79-91.

Hassenstern, B., 1960. Die bisherige Rolle der Kybernetik in der biologischen Forschung. Naturw. Rdsch. Jg. 13, Heft 9-11, 1-24.

Hensel, H., 1955. Mensch und warmblïtige Tiere. In: Temperatur und Leben. Springer, Berlin, Göttingen, Heidelberg, pp. 329-466.

HoLzER, H., 1961. Wirkungsmechanismus der Zytostatika. Dtsch. med. J. 12, 312-317. 
- 1963. Intrazelluläre Regulation des Stoffwechsels. Naturwissenschaften. Jg. 50, 260-270.

JACOB, F. \& MONOD, J., 1961. On the regulation of gene activity. Cold Spr. Harb. Symp. quant. Biol. 26, 193-212.

JUNGSTAND, W., 1962. Untersuchungen über die Neurosekretion und deren Abhängigkeit ron verschiedenen Außenfaktoren bei der Lungenschnecke Helix pomatia L. Zool. Jb. allgem. Zool. 70, 1-23.

KorringA, P., 1957. Water temperature and breeding throughout the geographical range of Ostrea edulis. Ann. biol., Paris, 3. ser. 33, 1-17.

LasizwsKr, R. C., 1963. Oxygen consumption of torpid, resting, active and flying humming birds. Pbysiol. Zoöl. 36, 122-140.

Loosanoff, V. L. \& Davis, H. C., 1952. Repeated semiannual spawning of northern oysters. Science 115, 675-676.

Lynen, F., Hartmann, G., Netter, K. F. \& Schuegraf, A., 1959. Phosphate turnover and Pasteur effect. Ciba Foundation Symp. Regulation of Cell Metabolism. J. \& A. Churchill, London, pp. 256-273.

McLean, F. C., 1960. Introduction: Homeostasis in Mineral Metabolism. In: Mineral Metabolism (edited by C. L. Comar \& F. Bronner), vol. I, Part A, Academic Press, New York and London, pp. 1-10.

Mittelstaedt, H., 1954. Regelung in der Biologie. Regelungstech. 2, 177-181.

- 1961. Die Regelungstheorie als methodisches Werkzeug der Verhaltensanalyse. Naturwissenschaften 48, 246-254.

Monod, J., 1956. Remarks on the mechanism of enzyme induction. In: Units of Biological Structure and Function. Academic Press, New York and London, pp. 7-28.

Morgan, A. H. \& Fales, C. H., 1942. Seasonal conditions and effects of low temperatures in the thyroid glands of amphibians. J. Morph. 71, 357-387.

Munday, K. A. \& Thompson, B. D., 1962. The effect of osmotic pressure on the activity of Carcinus maenas mitochondria. Comp. Biochem. Physiol. 6, 277-288.

Orton, J. H., 1920. Sea-temperature, breeding and distribution in marine animals. J. Mar. biol. Ass. U. K. 12, 339-366.

Peiss, C. N. \& FIELd, J., 1950. The respiratory metabolism of excised tissues of warm- and cold-adapted fishes. Biol. Bull., Woods Hole 99, 213-224.

Precht, H., 1951. Der Einfluß der Temperatur auf die Atmung und einige Fermente beim Aal (Anguilla vulgaris L.). Biol. Zbl. 70, 71-85.

- 1955. Wechselwarme Tiere und Pflanzen. In: Temperatur und Leben. Springer, Berlin, Göttingen, Heidelberg, pp. 1-177.

Prosser, L. C., Editor, 1950. Comparative Animal Physiology. W. B. Saunders Co. Philadelphia and London, $888 \mathrm{pp}$.

Ranke, O. F. \& KeIDel, W. D., 1960. Physiologie des Zentralnervensystems vom Standpunkt der Regelungslehre. Urban \& Schwarzenberg, München-Berlin, $133 \mathrm{pp}$.

Roberts, J. L., 1961. The influence of photoperiod upon thermal acclimation by the crucian carp, Carassius carassius L. Zool. Anz. 24 (Suppl.), 73-77.

Roots, B. I. \& Prosser, C. L., 1962. Temperature acclimation and the nervous system in fish. J. exp. Biol. 39, 617-630.

Rosenblueth, A., Wiener, N. \& Bigelow, J., 1943. Behaviour, Purpose and Teleology. Philosoph. Sci. 10.

SCHLIEPER, C., 1952. Versuch einer physiologischen Analyse der besonderen Eigenschaften einiger eurythermer Wassertiere. Biol. Źbl. 71, 449-461.

Scholander, P. F., FlagG, W., Walters, V. \& Irving, L., 1953. Climatic adaptation in arctic and tropical poikilotherms. Physiol. Zoöl. 26, 67-92.

SMITH, R. E., 1956. Quantitative relations between liver mitochondria metabolism and total body weight in mammals. Ann. N. Y. Acad. Sci, 62, 403-422.

STRANGWAYs-Dixon, J., 1961. The relationship between nutrition, hormones and reproduction in the blowfly Calliphora erythrocephala (MerG.). I. J. exp. Biol. 38, 225-235.

UMBARGER, H. E., 1956. Evidence for a negative-feedback mechanism in the biosynthesis of isoleucine. Science 123, 848. 
VALÈN, E., 1958. Oxygen consumption in relation to temperature in some poikilotherms. Acta physiol. scand. 42, 358-362.

WIENER, N., 1948. Cybernetics. The Mass. Inst. Technol. Press, 165 pp.

WIESER, W., 1963a. Parameter des Sauerstoffyerbrauches. II. Z. vergl. Physiol. 47, 1-16.

- 1963b. Die Organisation der Lebewesen als technisches Problem. Mathematik, Technik, Wirtschaft, Wien, Jg. 10, 17-22.

\section{Diskussion im Anschluß an den Vortrag WIESER}

LOCKER: Nachdem Herr WIEsER in seinem ausgezeichneten Vortrag einige Dinge vom Standpunkt der Regelkunde betrachtet hat, die ich selbst vom Gesichtspunkt der Theorie offener Systeme bzw. der Theorie des Fließgleichgewichts darzustellen versucht habe, wäre vielleicht schon ein detaillierter Vergleich zwischen beiden Betrachtungsweisen ein Thema für unser nächstes Symposion im Jahre 1965.

SMrTh: A key operator in the scheme which Dr. WIESER has presented might well be the concentration or production rate of TPN-TPNH. This is contingent upon (1) the fact that reduction of TPN occurs at only 5 or 6 metabolic steps which appear largely under thyroid hormonal control, and (2) the presence on each major pathway of at least one metabolic step which requires 'TPNH for its completion. Thus control through this co-factor may be on the main line of regulatory expression (c. f. Smith \& Foljer 1962; Physiol. Rev. 42, 60). Of interest here is also the recent demonstration by TATA et al. $(1963$; Biochem. J. 86, 408) that thyroid hormone in vivo in rats induces changes in metabolic rate, which appears contingent upon earlier activity involving protein synthesis.

WIESER: The main point I wanted to make was that the functioning of very complicated systems can be controlled by single key processes. Such a key position in metabolizing systems is held by the ATP/ADP link, and also by the TPN/TPNH link. You are quite correct in pointing out the importance of the latter system. The problem is of course that theoretically we know that these are important control points. However, it would be comforting to know whether the systems involved actually do have the characteristics of controlling systems. For example, under the influence of environmental or systemic stimuli, they would have to change in concentration or in turnover rate, and there must be a regulating centre which keeps this portion of the machinery going.

SMrTH: I think that the most exciting thing I have seen in this year has been the implications that can be drawn from the work of TATA et al. (loc. cit.), who have rather dramatically shown that thyroid hormone not only stimulates metabolism, but that its stimulation of metabolism appears to be contingent upon the synthesis of certain enzymes, all of which have not been specified. However, it gives us a very definite suggestion now that we may have some operators here at the level of the DNA to RNA at messenger RNA level. (These implications of this work were first drawn to my attention during the spring of 1963 by Mr. Hirtueman, a graduate student in our laboratory.) I think this is the inductive level at which metabolism will be accounted for in this present period and which may lead us to some understanding of hormonal action at a higher level of the integrated system. 